Corrigendum

\title{
Corrigendum to "EUSOMA position regarding breast implant associated anaplastic large cell lymphoma (BIA-ALCL) and the use of textured implants" [Breast 44 (April 2019) 90-93]
}

\author{
M.J. Cardoso ${ }^{\text {a, }}{ }^{*}$, L. Wyld $^{\text {b }}$, I.T. Rubio ${ }^{\text {c }}$, M. Leidenius ${ }^{\text {d }}$, G. Curigliano ${ }^{\text {e, }}$, B. Cutuli ${ }^{\mathrm{g}}$, \\ L. Marotti $^{\text {h }}$, L. Biganzoli ${ }^{\text {i }}$
}

${ }^{a}$ Breast Unit, Champalimaud Clinical Center/Champalimaud Foundation, Nova Medical School, Lisbon, Portugal

b Department of Oncology and Metabolism, University of Sheffield, Sheffield, UK

${ }^{\mathrm{c}}$ Breast Surgical Oncology, Clínica Universidad de Navarra, Madrid, Universidad de Navarra, Spain

${ }^{\mathrm{d}}$ Breast Surgery Unit, Comprehensive Cancer Center Helsinki University Hospital, Helsinki, Finland

e IEO, European Institute of Oncology IRCCS, Italy

${ }^{\mathrm{f}}$ University of Milano, Milan, Italy

${ }^{g}$ Institut du Cancer Courlancy, Reims, France

${ }^{\mathrm{h}}$ EUSOMA, Florence, Italy

i Breast Unit, Nuovo Ospedale di Prato, Prato, Italy

The authors regret that Prof. Curigliano's affiliations were not correct in the original version.

The authors would like to apologise for any inconvenience caused.

DOI of original article: https://doi.org/10.1016/j.breast.2019.01.011.

* Corresponding author.

E-mail address: maria.joao.cardoso@fundacaochampalimaud.pt (M.J. Cardoso). 